\title{
Incontinencia urinaria oculta evidenciada por estudio urodinámico preoperatorio en pacientes con prolapso genital severo
}

\author{
Ricci Arriola P, Solà Dalenz V, Pardo Schanz J. \\ Unidad de Uroginecología, Departamento de Ginecología, Clínica Las Condes.
}

Actas Urol Esp. 2008;32(8):827-832

\section{RESUMEN}

INCONTINENCIA URINARIA OCULTA EVIDENCIADA POR ESTUDIO URODINÁMICO PREOPERATORIO EN PACIENTES CON PROLAPSO GENITAL SEVERO

Objetivos: Revisar la eficacia de la urodinamia monocanal realizada con corrección del prolapso genital severo, mediante valva de Bresky, en la pesquisa de incontinencia de orina oculta, como prueba prequirúrgica, para planificar una corrección mediante cinta submedio uretral concomitantemente con la cirugía de prolapso.

Paciente y Método: Pacientes ingresadas a la Unidad de Uroginecología de Clínica Las Condes, entre enero del 2006 y diciembre del 2007, debido a cistocele grado III o IV. Pacientes sin antecedentes de cirugía de prolapso o incontinencia de orina. Se realiza una urodinamia monocanal con corrección del prolapso mediante una valva de Bresky. Se realiza medición de presión de retro-resistencia uretral y cistometría. En aquellas mujeres en que se descarta la presencia de incontinencia oculta, se realiza corrección del prolapso mediante malla Prolift. En aquellas en que se encuentra incontinencia oculta, se coloca concomitantemente, una cinta sub-medio uretral profiláctica, para evitar la aparición de incontinencia de orina en el postoperatorio.

Resultados: De las treinta y ocho mujeres que cumplieron el criterio de ingreso a este estudio, 14 (36,8\%) no registraron presencia de incontinencia de esfuerzo oculta durante la urodinamia. En las $24(63,2 \%)$ restantes se evidenció incontinencia de orina de esfuerzo. Tipo I, en 1; tipo II en 12; tipo III en 1; tipo II+III en 10. Durante la cistometría 3 de ellas registraron un detrusor hiperactivo asintomático. En 1 $(4,2 \%)$ de las 24 pacientes sometidas a cirugía profiláctica de incontinencia, se registró falla, con escapes de orina durante el postoperatorio inmediato. El resto (23) no presentó incontinencia durante el postoperatorio inmediato, temprano, ni tardío, completando un período de observación entre 4 y 24 meses. En las 14 mujeres con cirugía sólo para el prolapso, tampoco se registró aparición de incontinencia.

Conclusiones: La urodinamia con corrección del prolapso genital severo, mediante valva de Bresky, es un método eficiente en la detección de incontinencia de orina oculta. Lo que nos permite planificar una posible cirugía anti-incontinencia profiláctica. Que según nuestra experiencia, es un método seguro y eficaz. De esta forma, se entrega una solución integral, a un problema del suelo pelviano que frecuentemente se asocia, por compartir los mismos factores de riesgo y que significa un alto grado de deterioro de la calidad de vida.

Palabras clave: Prolapso genital. Incontinencia urinaria. Incontinencia oculta. Urodinamia.

\section{ABSTRACT}

\section{OCCULT STRESS INCONTINENCE IDENTIFY BY PREOPERATIVE URODYNAMIC STUDY IN WOMEN WITH SEVERE PELVIC ORGAN} PROLAPSE

Objective: To review the effectiveness of preoperative urodynamic study made with correction of the severe genital prolapse by Bresky valve in the diagnosis of urinary occult incontinence, in order to plan a correction with a prophylactic sub-mid urethral mesh in the prolapse surgery.

Patients and Method: Patients of the Urogynecology and Vaginal Surgery Unit of Las Condes Clinic, between January 2006 and December 2007, with grade III or IV cystocele. A condition was patients without previous prolapse and/or incontinence surgeries. A non multichannel urodynamics test was made with prolapse correction by a Bresky valve. The urethra retro resistance measurement and cystometry was made. In the women in whom the occult urinary incontinence was discards the prolapse surgery was made with Prolift mesh. In the women with occult incontinence a prophylactic sub-mid urethral tape was applied in the prolapse surgery, to avoid the appearance of incontinence after surgery.

Results: Thirty eight women fulfilled the criterion to enter in this study, $14(36,8 \%)$ of them did not register occult urinary incontinence in the urodynamic test. In the $24(63.2 \%)$ with occult incontinence: Type I in 1; type II in 2; type III in 1 and type II+III in 10. During the cystometry 3 of them registered an asymptomatic hyperactive detrusor. In $1(4.2 \%)$ of the 24 patients with prophylactic incontinence surgery, in the immediate postoperative time a failure was observed. The rest 23 women did not present incontinence during the observation period between 4 to 24 months. In the 14 women only with prolapse surgery incontinence was not registered.

Conclusions: The preoperative urodynamic test with correction of severe genital prolapse by Bresky valve is an efficient method to detect the occult urinary incontinence. This allows planning a prophylactic incontinence surgery. According to our experience this method is safe and effective to avoid that the occult incontinence appears after a severe cystocele surgery repair and allows to given an integral solution to the pelvic floor problems that frequently sees associate, because they risk factors are the same to these conditions and both cause a high degree of deterioration of quality of life.

Keywords: Genital prolapse. Urinary incontinence. Occult incontinence. Urodynamic. 
$\mathrm{E}^{1}$ prolapso genital es una condición patológica que impacta negativamente la calidad de vida en la mujer. Puede presentarse hasta en un $50 \%$ de las multíparas ${ }^{1}$ y su incidencia aumenta con la edad ${ }^{2}$.

Por otro lado, la incontinencia de orina puede afectar a una de cada cuatro mujeres y también tiene efectos negativos sobre quienes la padecen. Se trata de mujeres que presenta malestar, por el cual cambian sus hábitos de vida, se sienten estigmatizadas, por lo que prefieren apartarse de la vida familiar y social, para evitar que los demás se den cuenta de su alteración ${ }^{3}$.

En 1997, Olsen ${ }^{4}$ demostró que el 11,1\% de las mujeres que alcanzan los 80 años habían sido operadas por prolapso o incontinencia de orina, o ambas condiciones, y además que el 29,2\% de ellas requirió una reoperación por recidiva.

Tanto el prolapso como la incontinencia de orina son desórdenes del suelo pelviano, que comparten los mismos factores de riesgo. En la práctica clínica observaremos que el daño de los tejidos de sostén, puede provocar prolapso e incontinencia de orina de esfuerzo. Este daño puede ocurrir por sobredistención, debilitamiento o ruptura por compresión.

Con el aumento de la sobrevida de la mujer, veremos cada día con mayor frecuencia que consultan por estas patologías.

Por todas las razones anteriores, no es infrecuente encontrar la asociación de prolapso e incontinencia de orina en nuestras pacientes ${ }^{5,6}$.

Cuando corregimos un prolapso genital severo, si previamente no hemos estudiado y considerado la posibilidad de aparición de incontinencia de orina, podemos encontrarnos con una patología del suelo pelviano parcialmente corregida. Ya que es probable que la paciente tenga una incontinencia de orina oculta, que sólo será evidente después de la cirugía de prolapso.

La aparición de una incontinencia de orina después de la corrección de un prolapso, puede ser muy frustrante para la paciente y el médico ${ }^{7}$.

Con el objetivo de demostrar la eficacia de la urodinamia monocanal realizada con corrección del prolapso genital severo, mediante valva Bresky, como prueba prequirúrgica en la pesquisa de incontinencia de orina oculta, presentamos una serie de pacientes.

\section{PACIENTE Y MÉTODO}

Se realizó un estudio prospectivo de pacientes ingresadas por Prolapso Genital Severo, a la Unidad de Uroginecología del Departamento de Obstetricia y Ginecología de Clínica Las Condes, entre enero del 2006 y diciembre del 2007.

Criterio de inclusión: Pacientes ingresadas por cistocele grado III o IV de POP-Q, sin evidencias clínicas de incontinencia de orina en la anamnesis y examen físico. Sin antecedentes de cirugía previa por prolapso genital o incontinencia de orina.

Método para objetivar la incontinencia oculta: A todas las pacientes seleccionadas según el criterio de inclusión, se les realizó una urodinamia con reducción del prolapso por medio de una valva vaginal de Bresky (Fig. 1).

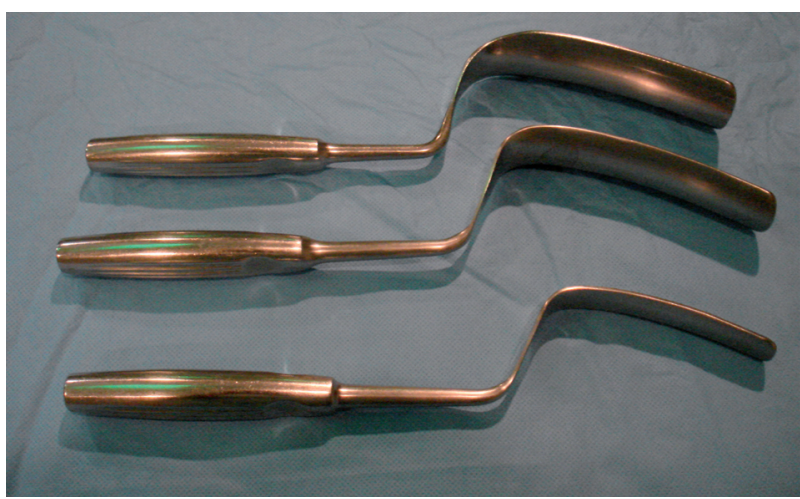

FIGURA 1. Valvas vaginales de Bresky de diferentes tamaños. Utilizadas en esta serie para reducir el cistocele severo durante la urodinamia, para evidenciar incontinencia de orina oculta.

Se utilizó el equipo de urodinamia monocanal con medición de retro-resistencia uretral y cistometría, MoniTorr (Gynecare, Worldwide, división de Ethicon INC, compañia de Johnson \& Johnson, Somerville, New Jersey) ${ }^{8}$ (Figs. 2A, 2B, 3A y 3B).

La presencia de incontinencia de orina de esfuerzo se objetivó por medio de la medición de presión de retro-resistencia uretral (PRU). Se utilizó la clasificación de McGuire et $\mathrm{al}^{9}$.

Además, se realizó cistometría para evidenciar la eventual presencia de detrusor hiperactivo asintomático.

Característica de las pacientes ingresadas al estudio: Se seleccionaron 38 pacientes entre enero del 2006 y diciembre del 2007. Las edades fluctuaron entre 30 y 90 años, con una media de 60 años. La paridad fluctuó entre 2 y 5 , con una media de 3 . 

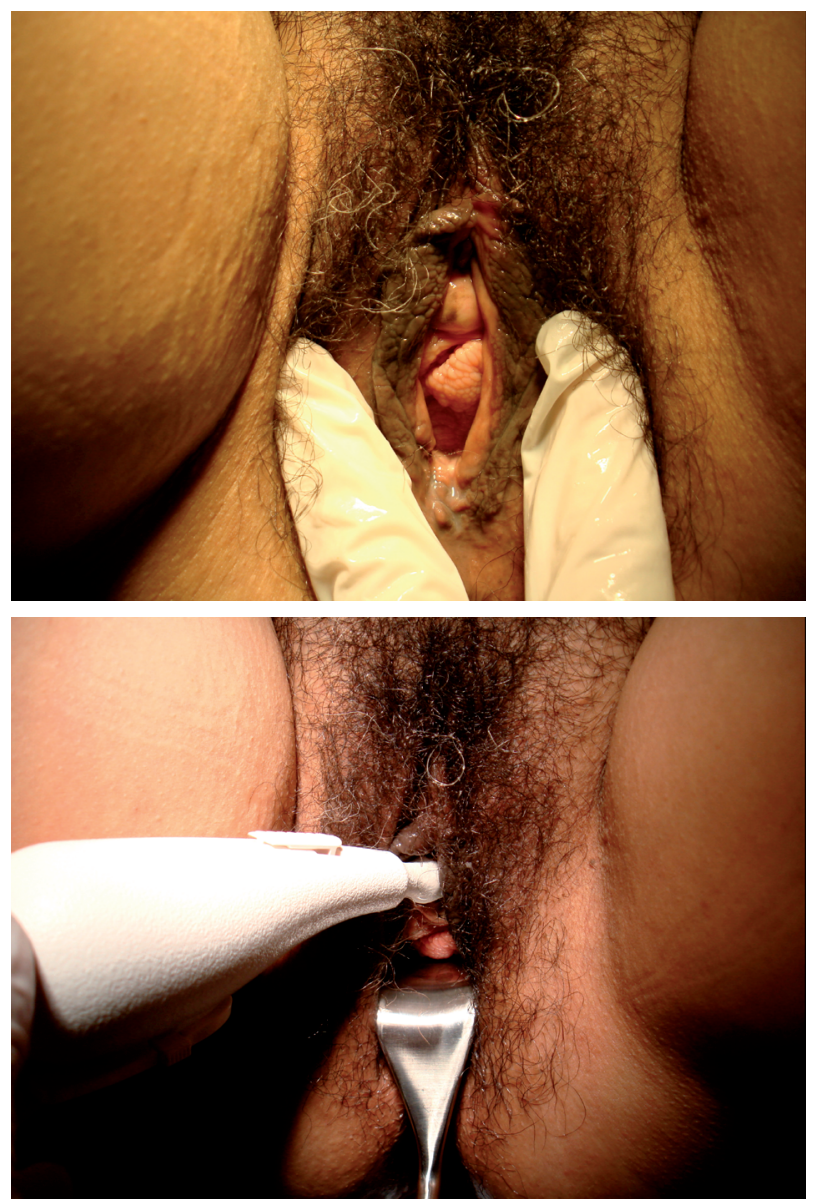

FIGURA 2. A) Pacientes con cistocele grado III de POP-Q. B) Medición de PRU (Presión de retro-resistencia uretral) con corrección del cistocele mediante valva de Bresky.

Las pacientes que demostraron tener una incontinencia de orina oculta, fueron operadas por el prolapso y además se les aplicó una cinta sub-medio uretral, tipo TVT-O o TVT-Secur, durante el mismo acto quirúrgico.

La corrección del prolapso genital grado III o IV se realizó mediante malla de anclaje Prolift (Johnson \& Johnson, Somerville, New Jersey) ${ }^{10}$.

Se completó un período de observación, para registrar la eventual aparición de incontinencia de orina en ambos grupos. Aquellas en que se corrigió sólo el prolapso, por descartarse la incontinencia oculta, y aquellas en que se realizó concomitantemente ambas cirugías, por demostrarse con urodinamia la presencia de incontinencia.

\section{RESULTADOS}

De las 38 pacientes, $14(36,8 \%)$ no registraron presencia de incontinencia de orina de esfuerzo oculta.
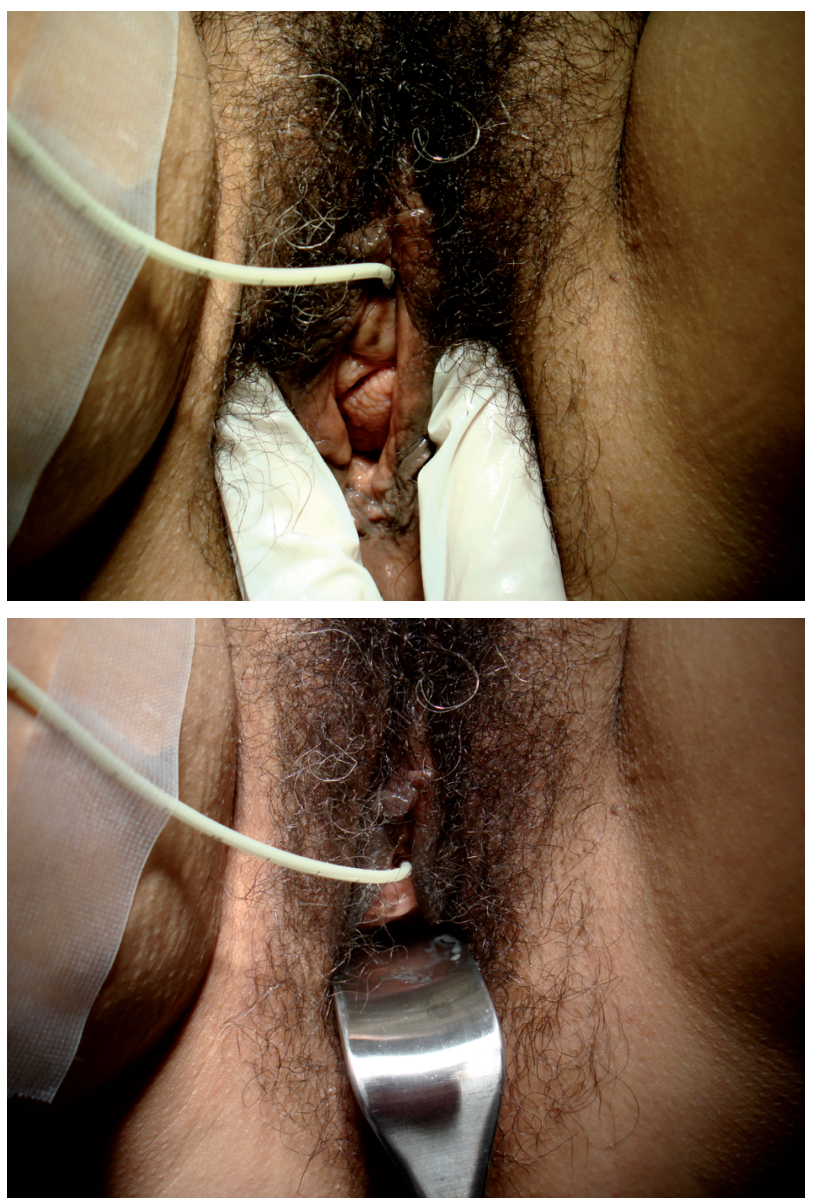

FIGURA 3. A) Cistometría sin corrección de prolapso.

B) Cistometria realizada con corrección del cistocele mediante valva vaginal de Bresky.

En las $24(63,2 \%)$ restantes se evidenció: incontinencia tipo I, en 1 paciente; tipo II en 12; tipo III en 1 y tipo II+III en 10.

Además, en 3 de las 24 se registró la presencia de un detrusor hiperactivo asintomático, al presentarse contracciones no inhibidas del detrusor durante la fase de llene vesical en la cistometría (Tabla 1).

Tabla 1. Resultado de la urodinamia con corrección del prolapso.

\begin{tabular}{lccc}
\hline & \multicolumn{2}{c}{ IOE* $^{*}$} & Asociación a DH \\
\hline Tipo & Número & \% & Número \\
\hline Normal & 14 & 36,8 & \\
I & 1 & 2,6 & \\
II & 12 & 31,6 & 1 \\
III & 1 & 2,6 & 1 \\
II + III & 10 & 26,3 & 1 \\
\hline Total & 38 & 100 & $3(7,9 \%)$ \\
\hline
\end{tabular}

* Clasificación de Incontinencia de Orina de Esfuerzo de McGuire et al. DH: detrusor hiperactivo. 
En $1(4,2 \%)$ de las 24 mujeres con incontinencia oculta, y cinta sub-medio uretral profiláctica, se registró una falla. Al observarse escapes de orina frente a los esfuerzos durante el postoperatorio inmediato. Correspondía a una paciente con registro preoperatorio de incontinencia de orina oculta tipo II+III. En ella se había utilizado una cinta TVTSecur profiláctica. Fue corregida exitosamente por medio de un TVT retropúbico clásico al mes de la primera cirugía.

En el resto de las mujeres de ambos grupos, con y sin cirugía profiláctica, no se presentó incontinencia durante el período de observación, que fue de 4 (1 mujer) a 24 meses, con una media de 12 .

No se registraron complicaciones asociadas a la anestesia, ni quirúrgicas durante el periodo intraoperatorio y postoperatorio inmediato o tardío.

\section{DISCUSIóN}

En otras experiencia publicadas, para evidenciar la presencia de incontinencia de orina oculta en pacientes con prolapso genital severo, se ha utilizado exitosamente la prueba del pesario ${ }^{11,13}$. Es decir, se corrige el prolapso mediante la introducción vaginal de un pesario, que se puede dejar por un tiempo, para su uso en el domicilio y demostrar posibles pérdidas de orina, mediante la recolección en apósitos. Sin embargo, también existen experiencias en las que se ha corregido la posición del cistocele con pesario, e inmediatamente se ha realizado urodinamia multicanal para certificar la presencia de la incontinencia, es decir similar a lo realizado en nuestra serie ${ }^{14,15}$.

La investigación urodinámica de pacientes que serán sometidas a cirugía por prolapso severo, permite evidenciar la presencia de incontinencia de orina oculta, y clasificar aquellas que ya son evidentes junto al cistocele ${ }^{16}$. De esta manera la urodinamia permite planificar estrategias óptimas de tratamiento, que comprenden una cirugía para corrección del prolapso y una cinta sub-medio uretral de acuerdo al tipo de incontinencia. La combinación de ambas cirugías en aquellas pacientes que lo requieren, permite resolver ambos problemas al mismo tiempo ${ }^{15}$.

El caso típico de pacientes que presentan prolapso genital severo, sin incontinencia de orina evidente, que después de la corrección del cistocele se puede hacer sintomático, por no realizar un estudio de simulación antes de la cirugía, es presentado en una publicación del 2007, por Yamada y Kobashi. En esta paciente se demostró la incontinencia con urodinamia y se corrigió con una cinta sub-medio uretral, en una segunda cirugía, que podría haber sido realizada en un solo tiempo, junto a la corrección del cistocele ${ }^{17}$.

En nuestras pacientes no utilizamos pesarios, ya que generalmente les incomoda el quedar con ellos por largo tiempo, en el caso de enviarlas a su domicilio con prueba de recolección de orina en apósitos. Por otro lado, generalmente vemos que en pacientes añosas deben conseguir ayuda para su inserción, no logrando una reducción completa del cistocele severo. Actualmente en nuestro país es difícil encontrar estos aparatos, ya que con el tiempo y advenimiento de terapias con carácter más definitivo, y poca preferencia por parte de las pacientes, se dejaron de comercializar. Por otro lado, el mejor y mayor acceso a técnicas quirúrgicas y nuevos medicamentos, nos ha permitido disminuir su prescripción. Por estas razones, buscamos un método más sencillo y alternativo para corregir el cistocele severo y así evidenciar una incontinencia de orina oculta por prolapso. La utilización de una valva vaginal resulta una forma sencilla y rápida, para corregir el prolapso mientras se realiza la urodinamia, que permite simular la condición de un cistocele corregido. Se trata de una simple valva, que es frecuentemente utilizada en todas nuestras cirugías vaginales. Las hay de diferente tamaño, y está siempre disponible en nuestro medio hospitalario. Sin perjuicio, que en otras experiencias pueda utilizarse cualquier tipo de valva que permita simular la corrección. Esto sigue el mismo principio de la "cirugía virtual" que propone la Teoría Integral de la Continencia, en que la corrección virtual de un defecto, ya sea por compresión o refuerzo mediante la aplicación de fuerzas o tensiones, que simulan lo que se realizará en la cirugía definitiva, nos permitirá evidenciar todos los posibles defectos a corregir, asegurando un mejor resultado quirúrgico posterior ${ }^{18,19}$. En este caso, con esta maniobra se hará evidente la incontinencia de orina si es que existe y permanece oculta debido al gran prolapso existente.

En muchos de los trabajos con corrección del cistocele severo por medio de pesarios, se evidencia la incontinencia oculta por medio de urodinamia multicanal. Esto permite objetivar y clasificar el tipo 
de incontinencia, para planificar la terapia definitiva más adecuada. Por esta razón, preferimos demostrar la presencia de incontinencia oculta, mediante la urodinamia, en este caso la monocanal con corrección del prolapso por una valva de Bresky durante el examen. La urodinamia monocanal nos permite demostrar de manera rápida, sencilla y objetiva, mediante valores numéricos, entregados por un examen que es menos invasivo y de menor costo que la urodinamia multicanal ${ }^{8}$.

Diferentes estudios han demostrado la presencia de incontinencia de esfuerzo oculta en mujeres con cistocele severo, en cifras que van desde el 30 al $80 \%{ }^{7,14,15,20,21}$. Nuestra serie la presentó en el $63,2 \%$.

Reena et al. demostraron en su serie que la posibilidad de encontrar incontinencia de orina oculta, aumenta al doble en pacientes mayores de 50 años $^{22}$. Esto no debe extrañarnos, ya que la incontinencia de orina de esfuerzo aumenta con la edad, lo que constituye un factor de riesgo también para prolapso.

Los factores de riesgo del prolapso genital son los mismos de la incontinencia de orina, lo que explica la alta asociación de ambas patologías. El mecanismo por medio del cual se evita que se exprese la incontinencia de orina en estas paciente, es la obstrucción uretral, provocada por el mismo prolapso severo ${ }^{7,9}$. Por esta razón, es importante distinguir aquellas pacientes en las que una vez que se corrija quirúrgicamente el prolapso, potencialmente comenzarán con incontinencia de orina. De esta forma al pesquisar antes de la corrección del prolapso, podremos planificar una cirugía anti-incontinencia concomitante ${ }^{23}$.

Brubaker et al. demostraron durante el 2006 que en pacientes sometidas a sacrocolpopexia por prolapso genital, se reducía la posibilidad de aparición de incontinencia de orina oculta, al realizar en forma concomitante una colposuspensión de Burch $^{24}$. Sin embargo, la mejor comprensión de la Teoría Integral de la Continencia, y el advenimiento de mejores cirugías del tipo cinta sub-medio uretral, ha permitido que hoy en día se utilicen preferentemente estas nuevas técnicas, que son más anatómicas y menos invasivas ${ }^{25}$. En casos de incontinencia oculta existen trabajos que demuestran efectividad con correcciones con técnica de $\mathrm{Burch}^{24}$, plicatura de la fascia endopélvica ${ }^{26} \mathrm{y}$ aplicación de TVT ${ }^{23,26,27}$. Por lo expuesto anteriormente, la mayoría son con cinta sub-medio uretral.
En la literatura médica, hoy podemos encontrar una gran variedad de trabajos que permiten asegurar la eficacia y seguridad de realizar cirugía con cinta sub-medio uretral en mujeres en las que se evidencia incontinencia de orina, con la reposición de prolapsos severos, como prueba antes de una corrección quirúrgica definitiva ${ }^{23,27-29}$. Incluso existen trabajos de aplicación de TVT en incontinencia urinaria de esfuerzo oculta, asociada a deficiencia intrínseca de esfinter ${ }^{29}$. El estudio de Meschia et al, demostró que en pacientes con incontinencia oculta, es más efectivo aplicar una cinta sub-medio uretral tipo TVT, que realizar una plicatura de la fascia endopélvica ${ }^{26}$. En nuestra serie se utilizó cinta submedio uretral tipo TVT-Secur o TVT-O. Y se presentó fracaso de la cirugía anti-incontinencia en un caso. El cual registró, aún cuando se asoció cirugía anti-incontinencia, inmediatamente en el postoperatorio, escapes de orina ante el esfuerzo. El resto de las mujeres con cinta sub-medio uretral no presentaron síntomas de incontinencia de orina en el postoperatorio inmediato, temprano, ni tardío, durante el período de seguimiento, con una media de 12 meses. Tampoco se encontró aparición de síntomas de incontinencia en las 14 mujeres sometidas sólo a corrección quirúrgica del prolapso, por haber descartado incontinencia oculta en la urodinamia preoperatoria con corrección del prolapso severo. En todo caso, esto no descarta que en el futuro presenten incontinencia, ya que como hemos planteado, los mismos factores son comunes para ambas condiciones patológicas, riesgo que aumenta además con la edad. Sólo el seguimiento a largo plazo y el reclutamiento de nuevas pacientes, en esta experiencia que continuará, nos permitirá definir el mantenimiento de estos buenos resultados en el tiempo. Protocolo de seguimiento que al momento de la comunicación de esta serie, con resultados preliminares, continúa.

En nuestra serie revisamos también la presencia de detrusor hiperactivo asintomático. La vejiga hiperactiva con o sin incontinencia de urgencia, se presenta en el 16,9\% de las mujeres mayores de 18 $a_{n}{ }^{30}{ }^{30}$. Por esto no es extraño haber encontrado su presencia en algunas de estas pacientes. Todas se encontraban asintomáticas y permanecieron igual después de la cirugía. Estas pacientes serán seguidas en forma especial, para evidenciar la posible aparición de síntomas en el futuro. 


\section{CONCLUSIÓN}

La urodinamia con corrección del prolapso genital severo, mediante valva vaginal de Bresky, es un método eficiente en la detección de incontinencia de orina oculta. Lo que nos permite planificar una posible cirugía anti-incontinencia, para evitar que durante el postoperatorio, se pueda hacer evidente una incontinencia previamente asintomática y potencial que no fue pesquisada. Se presenta la prueba de corrección de cistocele por medio de valva vaginal, como una alternativa a la realizada con pesarios.

Según nuestra serie, la aplicación de una cinta sub-medio uretral, concomitantemente a la corrección de prolapso genital severo, es un método seguro y eficaz en evitar que aparezca una incontinencia oculta.

La realización de ambas cirugías durante el mismo acto quirúrgico, permite dar una solución integral a un problema del suelo pelviano, que frecuentemente se ve asociado por compartir los mismos factores de riesgo y que significa un alto grado de deterioro de la calidad de vida.

\section{REFERENCIAS}

1. Carey MP, Dwyer PL. Genital prolapse: vaginal versus abdominal route of repair. Curr Opin Obstet Gynecol. 2001;13(5):499-505.

2. Mac Lennan AH, Taylor AE, Wilson DH, Wilson D. The prevalence of pelvic floor disorders and their relationship to gender, age, parity and mode of delivery. BJOG. 2000;107(12):1460-1470.

3. NIH State-of-the-Science Conference Statement on Prevention of Fecal and Urinary Incontinence in Adults. NIH Consens State Sci Statements. 2007;24(1).

4. Olsen AL, Smith VJ, Bergstrom JO, Colling JC, Clark AL. Epidemiology of surgically managed pelvic organ prolapse and urinary incontinence. Obstet Gynecol. 1997;89(4):501-506.

5. Brown JS, Waetjen LE, Subak LL, Thom DH, Van den Eeden S, Vittinghoff E. Pelvic organ prolapse surgery in the United States, 1997. Am J Obstet Gynecol. 2002;186(4):712-716.

6. Bradley CS, Kennedy CM, Nygaard IE. Pelvic floor symptoms and lifestyle factors in older women. J Womens Health (Larchmt). 2005: 14(2): 128-136.

7. Sinha D, Arunkalaivanan AS. Prevalence of occult stress incontinence in continent women with severe genital prolapse. J Obstet Gynaecol. 2007;27(2):174-176.

8. Solà Dalenz V, Ricci Arriola P, Pardo Schanz J. Estudio Urodinámico en la evaluación de la incontinencia urinaria femenina, con el nuevo sistema MoniTorr (Urodinamia Monocanal con medición de presión de retro-resistencia uretral). Experiencia con 100 pacientes. Actas Urol Esp. 2008;32(3):325-331.

9. McGuire EJ, Fitzpatrick CC, Wan J, Bloom D, Sanvordenker J, Ritchey M et al. Clinical assessment of urethral sphincter function. J Urol. 1993;150(5 Pt 1):1452-1454.

10. Solà Dalenz V, Pardo Schanz J, Ricci Arriola P, Guiloff Fische E. Sistema Prolift en la corrección del prolapso genital femenino. Actas Urol Esp. 2007;31(8):850-857.

11. Klutke JJ, Ramos S. Urodynamic outcome after surgery for severe prolapse and potential stress incontinence. Am J Obstet Gynecol. 2000;182(6): 1378-1381.

12. Liang CC, Chang YL, Chang SD, Lo TS, Soong YK. Pessary test to predict postoperative urinary incontinence in women undergoing hysterectomy for prolapse. Obstet Gynecol. 2004;104(4): 795-800.
13. Bhatia NN, Bergman A. Pessary test in women with urinary incontinence. Obstet Gynecol. 1985;65(2):220-226.

14. Chaikin DC, Groutz A, Blaivas JG. Predicting the need for antiincontinence surgery in continent women undergoing repair of severe urogenital prolapse. J Urol. 2000;163(2):531-534.

15. Roovers JP, Oelke M. Clinical relevance of urodynamic investigation tests prior to surgical correction of genital prolapse: a literature review. Int Urogynecol J Pelvic Floor Dysfunct. 2007; 18 (4):455-460.

16. Nguyen JN, Yazdany T, Burchette RJ. Urodynamic evaluation of urethral competency in women with posterior vaginal support defects. Urology. 2007;69(1):87-90.

17. Yamada BS, Kobashi KC. Urogenital prolapse and occult stress urinary incontinence. Nat Clin Pract Urol. 2007;4(1):55-58

18. Ricetto P, Palma P, Tarazona A. Aplicaciones clínicas de la teoría integral de la continencia. Actas Urol Esp. 2005;29(1):31-40.

19. Petros PE, Woodman PJ. The Integral Theory of continence. Int Urogynecol J Pelvic Floor Dysfunc. 2008;19(1):35-40.

20. Richardson DA, Bent AE, Ostergard DR. The effect of uterovaginal prolapse on urethrovesical pressure dynamics. Am J Obst Gynec 1983;146(8):901-905.

21. Rosenzweig BA, Pushkin S, Blumenfeld D, Bhastia NN. Prevalence of abnormal urodynamic test results in continent women with severe genitourinary prolapse. Obstet Gynecol. 1992;79(4):539-542.

22. Reena C, Kekre AN, Kekre N. Occult stress incontinence in women with pelvic organ prolapse. Int J Gynaecol Obstet. 2007;97(1):31-34.

23. Groutz A, Gold R, Pauzner D, Lessing JB, Gordon D. Tension-free vaginal tape (TVT) for the treatment of occult stress urinary incontinence in women undergoing prolapse repair: a prospective study of 100 consecutive cases. Neurourol Urodyn. 2004; 23(7):623-625.

24. Brubaker L, Cundiff GW, Fine P, Nygaard I, Richter HE, Visco AG, et al. Abdominal sacrocolpopexy with Burch colposuspension to reduce urinary stress incontinence. N Engl J Med. 2006;354(15):1557 1566.

25. Palma P. A requiem to the Burch. Int Urogynecol J Pelvic Floor Dysfunct. 2007;18(6):589-590.

26. Meschia M, Pifarotti P, Spennacchio M, Buonaguidi A, Gattei U, Somigliana E. A randomized comparison of tension-free vaginal tape and endopelvic fascia plication in women with genital prolapse and occult stress urinary incontinence. Am J Obstet Gynecol. 2004;190 (3):609-613.

27. Gordon D, Gold RS, Pauzner D, Lessing JB, Groutz A. Combined genitourinary prolapse repair and prophylactic tension-free vaginal tape in women with severe prolapse and occult stress urinary incontinence: preliminary results. Urology. 2001; 58(4):547-550.

28. de Tayrac R, Gervaise A, Chauveaud-Lambling A, Fernandez H Combined genital prolapse repair reinforced with a polypropylene mesh and tension-free vaginal tape in women with genital prolapse and stress urinary incontinence: a retrospective case-control study with short-term follow-up. Acta Obstet Gynecol Scand. 2004;83(10): 950-954.

29. Clemons JL, Aguilar VC, Sokol ER, Sung VW, Myers DL. Suburethral sling treatment of occult stress incontinence and intrinsic sphincter deficiency in women with severe vaginal prolapse of the anterior vs posterior/apical compartment. Am J Obstet Gynecol 2005; 192(5): 1566-1572.

30. Stewart WF, Van Rooyen JB, Cundiff GW, Abrams P, Herzog AR, Corey R, et al. Prevalence and burden of overactive bladder in the United States. World J Urol. 2003;20(6):327-336.

Correspondencia autor: Dr. P. Ricci Arriola

Unidad de Uroginecología

Departamento de Ginecología, Clínica Las Condes.

Lo Fontecilla 441, Las Condes, Santiago, Chile

Tel.: 56-2-2104610

E-mail autor: pricci@clinicalascondes.cl / pricci@vtr.net

Información articulo: Original - Incontinencia urinaria femenina

Trabajo recibido: abril 2008

Trabajo aceptado: mayo 2008 